\title{
Requirements Engineering for Embedded Systems: The REPES Process
}

\author{
Tarcísio Pereira $^{12}$, Fernanda Alencar ${ }^{1}$, and Jaelson Castro ${ }^{1}$ \\ Universidade Federal de Pernambuco - UFPE ${ }^{1}$ \\ $\{t c p, j b c\} @ c i n . u f p e . b r$, fernanda.ralencar@ufpe.br \\ Instituto Federal do Sertão Pernambucano - IF-Sertão ${ }^{2}$
}

\begin{abstract}
Context] Requirements Engineering (RE) for Embedded Systems (ES) is challenging since it has unique properties that make it complex, expensive and error-prone compared with other software categories, such as information systems. Due to their complexity, the risk of undetected requirements errors and deficiencies increases considerably. [Goal] Thus, this paper presents a specific process for requirements development and management named REPES which is tailored for embedded systems. [Method] In this proposal, we used the results of a Systematic Literature Review (SLR), metamodel and RE standards to guide the development of the process. [Results] REPES has four main process areas, 24 sub-processes, and 89 actions. [Conclusion] We argue that the proposed process can help organizations in improving their requirements practices to support completeness and correctness of RE for ES.
\end{abstract}

Keywords: requirements engineering $\cdot$ embedded systems $\cdot$ process.

\section{Introduction}

Requirements engineering for embedded systems is challenging since it has unique properties that make it complicated, expensive and error-prone compared with other software categories, such as information systems. Examples of such unique properties include: (i) Embedded Software Systems (ESS) are usually tightly coupled to their physical environment, (ii) the context of ESS requires a comprehensive range of stakeholders with different expertise such as requirements, mechanical, and electrical engineers, (iii) the interaction interfaces are mostly hardware components, (iv) Hardware Requirements Specification (HRS) is as essential as Software Requirements Specification (SRS), and (v) most of the embedded software functions are performed regularly and repetitively, for example, monitoring the speed, altitude, and attitude of an aircraft every $100 \mathrm{~ms}$. The system can activate these periodic software functions, or they can be initiated from a process state or on the operating environment or a command from the operator's input panel. Hence, it is essential to concern about the initiator of a function. [4].

Broy et al. [1] stated that in ES domain, more than $50 \%$ of the problems are only detected when the system is delivered. However, the problems reported 
by the customers are not related to the correctness of implementation but with requirements misconceptions. Hence, a RE process is crucial to reduce time, cost and improve quality goals [11].

Despite the number of studies considering RE in the ES domain, some issues remain such as (i) the lack of a particular RE process to guide academics/practitioners on how to apply their efforts systematically to meet requirements goals [10]. In order to fill this gap, we propose in this research a Requirements Engineering Process for Embedded Systems (REPES).

The goal of the process is to help organizations to improve and assess their requirements practices towards a more mature process execution. The process allows users to understand what should be done to achieve the desired results during its execution. Each sub-process has a purpose, a set of inputs, actions, and milestones. We used multiple information sources to collect data to define the practices to be included in the process, including one SLR [10], one metamodel [9], and a set of requirements standards.

This remainder of this paper is organized as follows: Section 2 presents the research goals. Ongoing work and contributions are presented in Section 3. Finally, conclusions and future works are shown in Section 4.

\section{Research Goals}

The core objective of this research is to provide a specific requirements engineering process for embedded systems, called REPES. In doing so, academics and practitioners can use it as a guide to improve their RE practices. In order to achieve the research objective, we defined the following research questions:

- RQ1. What is the state of the art on requirements engineering for embedded systems?

- RQ2. What should be taken into account in the development of requirements engineering for embedded systems?

- RQ3. How to derive RE actions/practices to compose the requirements process?

- RQ4. How should the feasibility of the new requirements engineering process be validated?

\section{Ongoing Work and Contributions}

To achieve the goal of this research, we have followed 6 steps to develop the requirements process.

1) Knowledge acquisition (RQ1): In this first step, we investigated the literature available about RE for embedded systems to become familiar with the domain. In order to accomplish this goal, we performed a Systematic Literature Review [10] to evaluate and synthesize the evidence available in the literature. We answered research questions related to the use of approaches, methods, techniques, and processes to support the RE in the ES domain. 
2) Problem definition: After answering a set of questions regarding RE for embedded systems, we noticed the lack of a requirements engineering process for embedded systems. Hence, we conclude that there is a need for a specific process for this domain. This issue lead us to another problem, (ii) what should be considered when developing such RE process?

3) Metamodel development (RQ2): To overcome above question mentioned, we developed a metamodel [8] that captures ES concepts with their interrelations. The metamodel was recently revised [9].

4) Identification of information sources (RQ3): In this step, we took the concepts of the metamodel and looked for them in the studies of the SLR as well as the main RE standards. In doing so, we were able to identify and select the information sources for the requirements engineering sub-processes as well as actions/practices. The following RE standards were considered: IEEE Std 1233:1998, IEEE Std 830:1998, ISO/IEC 12207, ISO/IEC 29158, ISO/IEC 15289, ISO/IEC 15288, INCOSE Handbook, SE-CMM, and CMMI-DEV.

5) Definition of process design (RQ3): After the analysis of the information sources, we established the design of the process. We followed the structure of Uni-REPM since it is a universal lightweight model to evaluate the maturity of a RE process [12].

The model hierarchy has three levels, namely: Main Process Area (MPA), Sub-Process Area (SPA) and Action. On the top level of the model, there are four Main Process Areas (Business Requirements, System Requirements, Software and Hardware Requirements, and Security Requirements). Each MPA is further broken down into several SPAs. On the bottom level, an Action denotes a particular activity that should be executed or a specific item that should be present.

6) Development of the process (RQ3): In this step, we developed the process. It has 4 process areas, 24 sub-processes, and 89 actions.

The process steps are not meant to be strictly sequential, apart from the first step; they are only given for guidance. A summary of the REPES process is presented in Figure 1.

Now, we need to evaluate the process. Hence, we are planning to conduct a survey with domain experts to find out whether the knowledge in literature was reasonably transferred and presented in the process [6]. Additionally, we intend to perform a case study applying the Goal Question Metric (GQM) measurement method to evaluate the feasibility of the proposed process in industry (RQ4).

According to [2], experts can determine the most relevant information for a given context, structuring the problem definition, and finding an appropriate solution. Thus, the survey will be conducted with at least 5 potential candidates. They should have fully developed and internalized skills and knowledge, including an ability to contribute new knowledge or technology. Therefore, we will use the guidelines provided by [7] to select the appropriate experts.

The goal of the evaluation is to answer the following research question: "Is the REPES process viable, complete and adequate?". In this sense, the questionnaire will be related to issues like experts role in industrial projects; the way process 


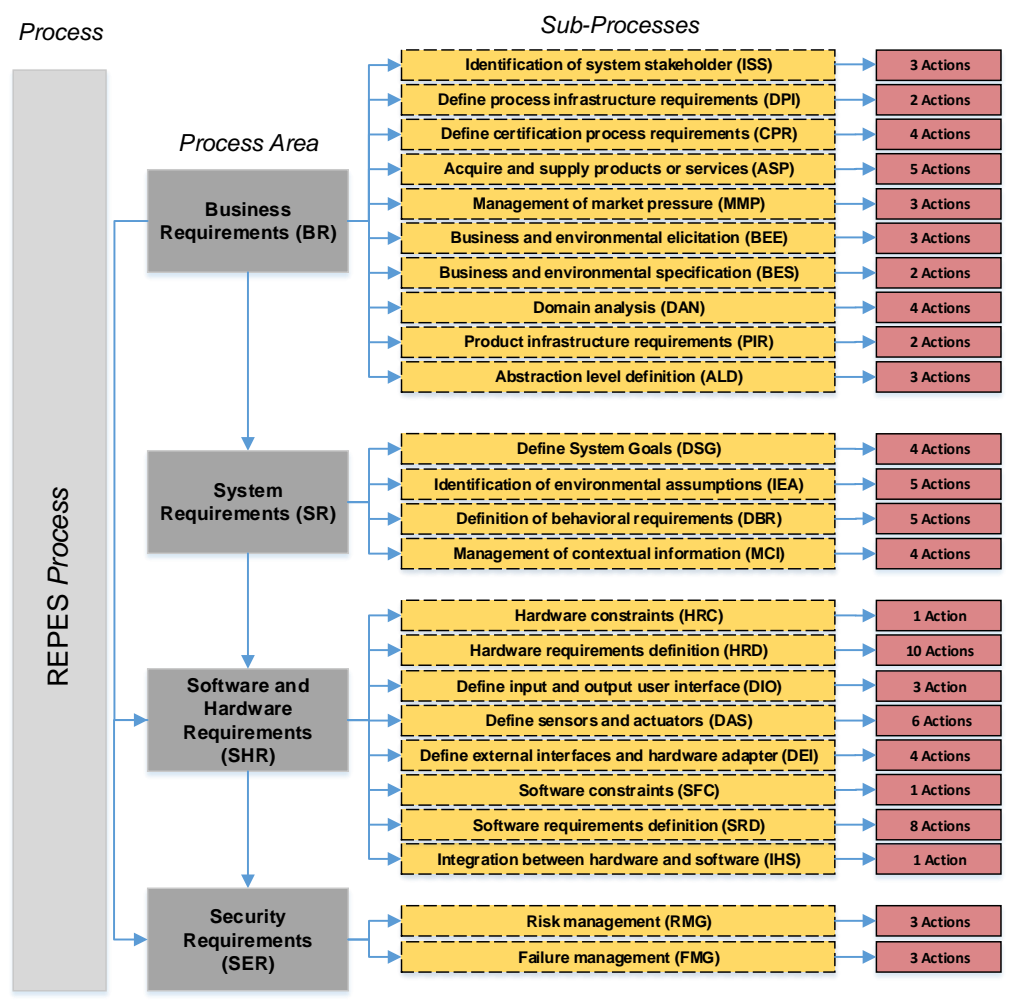

Fig. 1. REPES process representation.

areas are distributed; the specification, description and primary goal of the process areas; goals related to the sub-processes; inputs, actions, and work products associated to the goals/sub-processes; possible gaps in the evolution prescribed by each sub-process; and, the difficulty in evolving through the process areas;

The survey with experts will provide early feedback that will help to identify potential problems without using industry resources [6]. Thus, we can improve the proposed process for the next evaluation.

After the application of the survey with domain experts, we expect to perform a case study to evaluate the REPES process regarding its implementation feasibility. Feasibility, in this case, is defined by the completeness and adequacy of the process, and its elements, in the embedded systems industry. We will use the GQM method to describe the goal of our evaluation and derive a set of questions that should be answered to determine whether the goal was achieved or not. The goal of this evaluation is to Analyze the REPES process (object) for the purpose of assessment (purpose) with respect to feasibility (quality focus) 
from the point of view of the practitioner (viewpoint) in the context of embedded systems development companies (environment).

The questions will be derived from the assessment dimensions of the Motorola assessment instrument (approach, deployment, and results), which has the goal of evaluating an organization's current status about the Capability Maturity Model (CMM). It identifies weak areas for immediate attention and improvement [3]. The metrics will be defined following the steps used in the RE Maturity Measurement Framework (REMMF) to measure the maturity of requirements engineering processes [5].

When finished, this research will contribute to:

Systematic Literature Review. The identification of the state of the art on requirements engineering for embedded systems [10]. Obtained through a SLR.

Metamodel for Embedded Systems. Based on the SLR, we developed a metamodel that depicts information about the central concepts involved in the development of ES [8, 9].

Requirements Engineering Process for Embedded Systems. We will rely on the results of the SLR, the metamodel concepts, and on requirements engineering standards, to develop a Requirements Engineering Process for Embedded Systems called REPES [9].

[ONGOING] Evaluation of REPES. Two evaluations will be conducted to check completeness, correctness, feasibility, and adequacy of the REPES.

[ONGOING] Development of a Tool. Development of a tool to support the application of the REPES process.

\section{Conclusions and Future Work}

According to our SLR [10], the embedded systems community does not have a well-defined, standardized, and known requirements engineering process to guide companies to achieve a systematic RE level. Hence, there is a need to investigate and develop a specific requirements engineering process which will provide inputs, practices, and work products for requirements development and management for embedded systems.

Our requirements process will help requirements engineers to develop embedded systems with high quality. It could be used as a guide to assess the requirements activities of organizations. We also aim to help organizations to improve their maturity level.

We are currently planning to perform two evaluations of the REPES. The first one consists of a survey with experts to analyze the proposed process in order to (1) identify some gaps and mistakes in the process organization and specification; (2) evaluate the process areas, their goals and work products; and (3) assess the relationship between the process areas and the evolutional path through these areas. The second evaluation will be conducted with companies to evaluate the feasibility, completeness, and adequacy of the process in the industry. 


\subsection{Further Research}

This work has generated some research directions that should be explored in future efforts: (1) How to validate the usefulness and ease of use of the process? (2) What are the features of a software tool to support the REPES adoption should have? (3) How to develop a CASE tool to support the process? (4) What is the level of acceptance of REPES process by potential users? and (5) What is the REPES impact considering before/after assessment of RE practices in organizations?

Acknowledgments. The following Brazilian institutions have supported this work: UFPE, UPE, and IFSERTÃO-PE.

\section{References}

[1] Broy, M., Stauner, T.: Requirements engineering for embedded systems. Informationstechnik und Technische Informatik 41, 7-11 (1999)

[2] Chi, M.T.: Two approaches to the study of experts' characteristics. The Cambridge handbook of expertise and expert performance pp. 21-30 (2006)

[3] Daskalantonakis, M.K.: Achieving higher sei levels. IEEE software 11(4), 17-24 (1994)

[4] Mate, J.L., Silva, A.: Requirements Engineering for Sociotechnical Systems. Information Science Publishing (2005)

[5] Niazi, M., Wilson, D., Zowghi, D.: A maturity model for the implementation of software process improvement: an empirical study. Journal of Systems and Software 74(2), 155-172 (2005)

[6] Nikula, U., Sajaniemi, J., Kälviäinen, H.: A State-of-the-practice Survey on Requirements Engineering in Small-and Medium-sized Enterprises. Lappeenranta University of Technology Lappeenranta, Finland (2000)

[7] NUREG-1150: Reactor risk reference document,. In: Technical report (1989)

[8] Pereira, T., Albuquerque, D., Sousa, A., Alencar, F., Castro, J.: Towards a metamodel for a requirements engineering process of embedded systems. In: 2016 VI Brazilian Symposium on Computing Systems Engineering. pp. 93-100 (Nov 2016)

[9] Pereira, T., Sousa, A., Reinaldo, S., Albuquerque, D., Alencar, F., Castro, J.: A metamodel to guide a requirements elicitation process for embedded systems. accepted for publication. In: 11th International Conference on the Quality of Information and Communications Technology. IEEE (2018)

[10] Pereira, T., Albuquerque, D., Sousa, A., Alencar, F.M.R., Castro, J.: Retrospective and trends in requirements engineering for embedded systems: A systematic literature review. In: Proceedings of the XX Iberoamerican Conference on Software Engineering, Buenos Aires, Argentina, May 22-23, 2017. pp. 427-440 (2017)

[11] Sikora, E., Tenbergen, B., Pohl, K.: Industry needs and research directions in requirements engineering for embedded systems. Requirements Engineering 17(1), 57-78 (2012)

[12] Svahnberg, M., Gorschek, T., Nguyen, T.T.L., Nguyen, M.: Uni-repm: a framework for requirements engineering process assessment. Requirements Engineering 20(1), 91-118 (2015) 